\title{
Uncertainty clouds test of 'preemptive' therapy to ease autism traits
}

\section{BY PETER HESS}

24 SEPTEMBER 2021

Headlines this week hailed a behavioral therapy that parents can deliver to their babies to lessen the intensity of the children's autism traits and lower their chances of a diagnosis at age 3 , but independent experts urge caution: The observed effects were small, and the study's analysis raises questions.

"Even given the less-than-optimal statistical analyses and weaker-than-ideal outcomes, this is important, relevant and timely work," says April Benasich, professor of developmental cognitive neuroscience at Rutgers University in Newark, New Jersey, who was not involved in the research.

The study is a follow-up to a 2019 single-blind randomized controlled trial involving 103 babies who showed behavioral signs of autism at 9 to 14 months of age. Six months later, babies who had received the experimental therapy fared no differently than controls on a measure of autism traits.

In the new work, the same team followed 89 of the children to age 3. By then, 12 had been diagnosed with autism, and those in the control group, who had not received the experimental therapy, were three times as likely as those who had to meet diagnostic criteria for the condition, the study shows. The work appeared on Monday in JAMA Pediatrics.

"Although these effects were quite small in magnitude, with uncertain clinical significance," Benasich says, "it is still worth doing and, importantly, teaches the parents valuable skills in communicating with their children."

\section{Guiding interactions:}

The children in the study all screened positive for signs of autism on the Social Attention and Communication Surveillance-Revised measure at 9 to 14 months old. The tool is most accurate when administered several times, but many children take it only once before being referred to a 


\section{Spectrum | Autism Research News}

https://www.spectrumnews.org

specialist or not, says lead researcher Andrew Whitehouse, professor of autism research at the Telethon Kids Institute in Western Australia.

The parents of 50 babies learned to deliver the experimental therapy, called iBASIS-Video Interaction to Promote Positive Parenting (iBASIS-VIPP). During 10 biweekly sessions, the parents watched videos of their own interactions with their child, while a trained therapist offered them communication tips. The parents practiced their skills with their children for at least 15 minutes a day over the original five-month study period. The parents of another 53 children did not receive any training, but some received other therapies elsewhere.

The new work reassessed autism traits among 44 children from the control group and 45 children in the treatment group at ages 2 and 3, using the Autism Diagnostic Observation Schedule (ADOS). Clinicians also evaluated the children for autism at age 3.

Those whose parents had gone through training showed less pronounced autism traits than controls, when measured across the time period of 12 months to 3 years old. And three children in the treatment group received an autism diagnosis, compared with nine controls.

"The children falling below the diagnostic threshold still had developmental difficulties," Whitehouse says. "However, what this study showed was that by working with each child's unique differences, rather than trying to counter them, iBASIS-VIPP therapy has effectively supported their development throughout the early childhood years."

The results jibe with studies of the same therapy in younger siblings of autistic children - so-called 'baby sibs,' who are about 20 times as likely to have autism as the general population. After their parents were trained using iBASIS-VIPP, the baby sibs showed a significant reduction in autism traits at age 3 as measured by the ADOS or the Autism Observation Scale for Infants, a 2017 study found.

\section{Ethical issues:}

The study's primary outcome, trait severity differences, was statistically significant according to a one-tailed test, which examines only whether a treatment is more effective than the control condition. But the choice of a one-tailed test rather than a more stringent two-tailed one - which accounts for the possibility that a treatment may be either more or less effective than treatment as usual - boosted the odds that the findings would be statistically significant, Benasich says.

"That analytical choice precludes the opportunity to explore an effect in the other direction and, given the weak statistical results at one-tailed level, the likelihood is that the two-tailed results would not or did not reach significance," Benasich says.

Autism diagnoses at age 3, the study's secondary outcome, did meet conventional statistical 


\section{Spectrum | Autism Research News}

https://www.spectrumnews.org

significance thresholds for two-tailed testing, though a preregistered plan for conducting the study called for a one-tailed test, Whitehouse says.

The small number of participants also lessens confidence in the findings, says Regina Nuzzo, senior advisor for statistics communication at the American Statistical Association, who was not involved in the study.

Statistics notwithstanding, the investigation presents a "very rare" example of a research team doing everything in its power to evaluate an early autism intervention with minimal risks of bias, says Kristen Bottema-Beutel, associate professor of teaching, curriculum and society at Boston College in Massachusetts, who was not involved in the work.

"They checked all the boxes that researchers never check," she says. For instance, the team preregistered its hypotheses and detailed how they randomized the groups.

But the premise of the study - what Bottema-Beutel calls a preemptive intervention to keep people from becoming autistic - raises ethical concerns, she says. "Many autistic traits are just differences, they're not necessarily something that's going to make it more difficult for you to achieve developmental milestones."

The team is sensitive to this concern, Whitehouse says. "Our focus on supporting parent-child interactions is to support and enrich the social environment around the baby - creating learning opportunities for the baby that are tailored to the child's unique abilities."

He and his colleagues plan to continue following the children to see whether the effects detected in the study endure.

Cite this article: https://doi.org/10.53053/RXSS3973 